\title{
Magellanic Cloud planetary nebulae as probes of stellar evolution and populations
}

\author{
By LETIZIA STA NGHELLIN I
}

Space Telescope Science Institute, and European Space Agency

\begin{abstract}
Planetary Nebulae (PNs) in the Magellanic Clouds offer the unique opportunity to study both the population and evolution of low- and intermediate-mass stars, in an environment that is free of the distance scale bias and the differential reddening that hinder the observations of the Galactic sample. The study of LMC and SMC PNs also offers the direct comparison of stellar populations with different metallicity. The relative proximity of the Magellanic Clouds allows detailed spectroscopic analysis of the PNs therein, while the Hubble Space Telescope (HST) is necessary to obtain their spatially-resolved images. In this paper we discuss the history and evolution of this relatively recent branch of stellar astrophysics by reviewing the pioneering studies, and the most recent ground- and space-based achievements. In particular, we present the results from our recent HST surveys, including the metallicity dependence of PN identification (and, ultimately, the metallicity dependence of PN counts in galaxies); the morphological analysis of Magellanic PNs, and the correlations between morphology and other nebular properties; the relations between morphology and progenitor mass and age; and the direct analysis of Magellanic central stars and their importance to stellar evolution. Our morphological results are broadly consistent with the predictions of stellar evolution if the progenitors of asymmetric PNs have on average larger masses than the progenitors of symmetric PNs, without any assumption or relation to binarity of the stellar progenitors.
\end{abstract}

\section{Introduction}

Planetary Nebulae (PNs) are the gaseous relics of the envelopes ejected by low- and intermediate-mass stars $\left(1<M<8 \mathrm{M}_{\odot}\right)$ at the tip of the asymptotic giant branch (AGB), thus they are important probes of stellar evolution, stellar populations, and cosmic recycling. PNs have been observed in the Local Group (as well as in external galaxies), probing stellar evolution and populations in relation to their environment.

The details of the observations of Galactic PNs and their central stars (CSs) typically surpass the details of stellar and hydrodynamic models. Galactic PN studies are a necessary background toward the understanding the PN population in Local Group galaxies. Yet, the distance scale of Galactic PNs is uncertain to such a degree that the meaning of the comparison between observations and theory is hindered. By the same token, statistical studies of PN populations in the Galaxy suffer for the observational bias against the detection of Galactic disk PNs, and for the patchy interstellar extinction.

PNs in the Magellanic Clouds (LMC, SMC), hundreds of low-extinction planetaries at uniformly known distances, are a real bounty for the stellar evolution scientist. The composition gradient between the LMC, the SMC, and the Galaxy, afford the study of the effects of environment metallicity on PN evolution. The relative vicinity of the Clouds, and the spatial resolution that can be achieved with the Hubble Space Telescope (HST), allow the detection of PN morphology. Studying the PNs in the Magellanic Clouds is a perfect example of how the Local Group can be efficiently (and uniquely) used as an astrophysical laboratory. In this paper we review the history and the evolution of this field of study, with particular focus on the results from our recent HST Magellanic PN programs. 


\section{Pioneers in the field, and recent developments}

PN studies in the Magellanic Clouds are relatively recent. The first detection and spectral identification of Magellanic PNs is due to Lindsay (1955). Studies of Magellanic PN samples became common, and their importance evident, in the early sixties (Aller 1961; Westerlund 1964). Ground based observations of Magellanic PNs suffer from the fact that the contributions of nebular and stellar radiation are superimposed. Attempts to measure the CS magnitudes have been hampered by the difficulties in separating stellar and nebular contributions (e.g., Webster 1969).

Observations with the IUE, combined with the optical spectra acquired from the ground, have allowed the abundance analysis of Magellanic PNs. Space observations in the UV range were used for the detection of the complete set of carbon lines at various ionization stages, and made the carbon abundance derivation much more reliable. The key results in abundance studies can be found, to name a few, in Peimbert (1984), Boroson \& Liebert (1989), Kaler \& Jacoby (1991). Optical spectroscopy of large samples of Magellanic PNs have been carried out by Dopita and collaborators (Meatheringham \& Dopita 1991ab; Vassiliadis, Dopita, Morgan, \& Bell 1992). IUE observations were also used to measure the stellar luminosity beyond the Lyman limit for the CSs, giving an estimate of the total stellar luminosity, and and approximate estimate to the mass (Aller et al. 1987). Several papers on Magellanic PN spectroscopy, abundances, and the connection of nebular and stellar evolution can be found in the proceedings of IAU Symposia on planetary nebulae (e.g., Westerlund 1968; Feast 1968; Webster 1978), while the most recent, complete review on Magellanic PNs is due to Barlow (1989).

Studies of the chemical content of Magellanic PNs have been active in the recent past as well. On the observational side, Leisy \& Dennefeld (1996), and Costa, de Freitas Pacheco, \& Idiart (2000), have estimated new chemical abundances for several Magellanic PNs from optical and UV observations, enriching the databases for studies on the dredgeup of post-AGB stars and on the ISM enrichment in galaxies. On the theoretical side, van den Hoek \& Groenewegen (1997) calculated new chemical yields of the interstellar medium enrichment from synthetic evolution of intermediate-mass stars. With models from a wide range of initial masses and metallicities (included those appropriate for the Magellanic populations), van den Hoek \& Groenewegen confirm that the yields of nitrogen and carbon change abruptly for $\mathrm{M}>3 \mathrm{M}_{\odot}$, due to the hot-bottom burning effect.

To date, 277 LMC PNs (Leisy, Dennefeld, Alard, \& Guibert 1997) and 55 SMC PNs (Meyssonnier \& Azzoppardi 1993) are known. The total number of Magellanic PNs have more than doubled from the last count by Barlow (1989). In the last few years, several emission line surveys have been completed, or are near completion (e.g., UKST survey: Morgan 1998, Parker \& Phillips 1998; UM/CTIO survey: Smith et al. 1996). Future analysis of these surveys is essential for the future health of Magellanic PN research. We expect that the PN counts in the Clouds will increase significantly, improving the statistical significance of these studies. One important aspect of these surveys is the discovery of fainter PNs, that contributes to increasing the reliability of the faint end of the Magellanic PN luminosity function, and to enlarge the pool of known evolved PNs.

Related to the populations of Magellanic PNs is the 2MASS survey (Egan, Van Dyk, \& Price 2001). The importance of this multi-wavelength infrared survey to LMC PNs is related to the spatial distribution of the different types of AGB stars. Egan et al. showed that low mass AGB stars occupy the whole of the LMC projected volume, while the higher mass, younger population, AGB stars populate preferentially the LMC bar. Chemical and morphological studies of large LMC PN samples should be compared to the 
TABLE 1. STIS observations of Magellanic PNs

$\begin{array}{lllll}\text { Program } & \text { Galaxy } & \text { PNs } & \text { Observing mode } & \text { Papers }^{a} \\ & & & & \\ 8271 & \text { LMC } & 29 & \text { G750M/G430M/50CCD } & \text { I, II } \\ 8663 & \text { SMC } & 29^{b} & \text { G750M/G430M/50CCD } & \text { III, IV } \\ 9077 & \text { LMC } & 52 & \text { G750M/G430M/50CCD } & \text { V } \\ 9120 & \text { LMC } & 28 & \text { G140L/G230L } & \text { VI }\end{array}$

${ }^{a}$ Papers V and VI are in preparation; ${ }^{b}$ Two PNs in this sample are misclassified H II regions.

AGB samples, to relate the PN populations in the LMC to their immediate evolutionary progenitors.

\section{Early Hubble Space Telescope observations}

Extended studies of Galactic PNs have shown that PN morphology is intimately related to the mass and evolution of their CSs, to their stellar progenitors, and to the nebular chemistry. In the case of the LMC and the SMC, morphological studies became possible with the use of the cameras on the HST. The HST has also the capability of spatially separate the image of the nebula and that of the CS, making direct stellar analysis possible.

The early narrow-band images of Magellanic PNs were obtained before the first HST servicing mission (i.e., before the installation of COSTAR on HST) with the Faint Object Camera (Blades et al. 1992). Other images by Blades et al. have later been published by Stanghellini et al. (1999), where the quality of the pre-COSTAR images was validate through their comparison with post-COSTAR images of the same objects. These papers have made available 15 Magellanic PN images usable for statistical and morphological studies, while another 15 LMC PNs have been observed with the Planetary Camera 1 by Dopita et al. (Dopita et al. 1996; Vassiliadis et al. 1998). Finally, an additional ten Wide Field and Planetary Camera 2 narrow-band images of LMC PNs are available in the Hubble Data Archive (program 6407).

\section{Our Magellanic PN program}

During the HST Cycle 8 we started a series of surveys aimed at obtain the size, morphology, and CS properties of all Magellanic PNs known to date. The HST was an obligatory choice, since the Magellanic PNs are typically half an arcsec across, thus they are generally not resolved with ground-based telescopes.

The medium-dispersion, slitless capability of STIS offers us a valuable opportunity to study the evolution and morphology of the Magellanic Cloud PNs and their CSs at once. We have applied this capability in several SNAPSHOT surveys, obtaining images in the light of up to 7 of the most prominent low- and moderate-ionization optical, nebular emission lines. We also obtained direct continuum images to identify the correct CS (in spite of the crowded fields), and to measure the optical continuum emission.

In addition to the optical slitless spectra and broad band continuum images of the LMC and SMC PNs, we have acquired STIS UV spectra of 24 LMC PNs. In the cases where the CSs were hard to find in our STIS broad band images, we have also acquired WFPC2 Strömgren images (Program 8702). We have used through our investigations the 

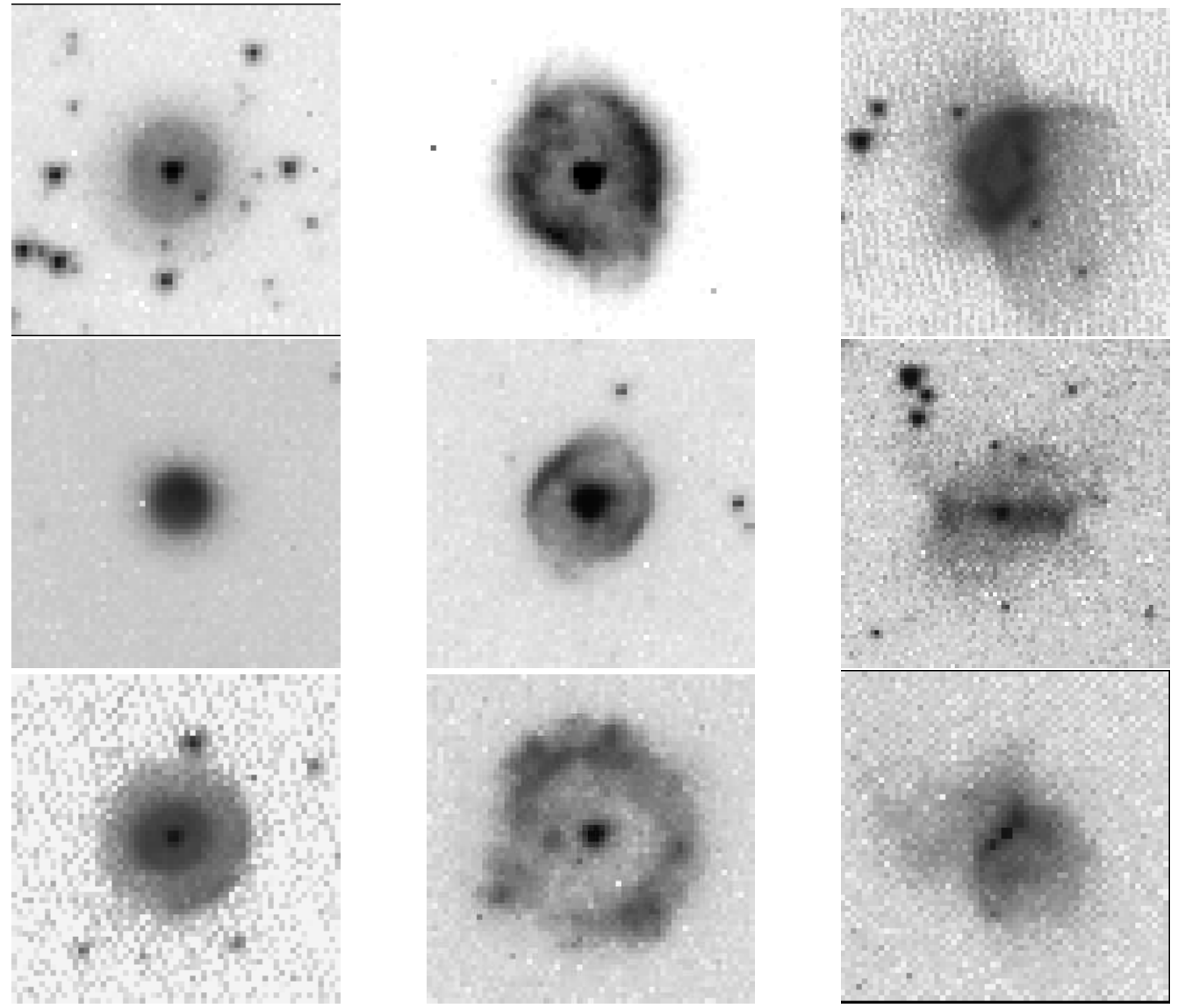

Figure 1. Magellanic PNs. Left panel: round PNs LMCJ 33, SMC-SP 34, and LMC-MG 40; Central panel: elliptical PNs LMC-SMP 101, SMC-MG 8, and SMC-MG 13; Right panel: bipolar PNs LMC-SMP 91, SMC-MA 1682, and LMC-MG 16. All thumbnails are $9 \operatorname{arcsec}^{2} \operatorname{sections}$ the STIS broad-band images.

limited data available in the literature (see Stanghellini et al. 1999). In Table 1 we list the major HST data sets of our Magellanic PN project. The actual data can be easily retrieved from the dedicated MAST Archival page http://archive.stsci.edu/hst/mcpn. In addition to the $H S T$ data we have made extensive use of the spectra acquired from the ground by us (papers in preparation), and available in the literature.

In Figure 1 we show a sampler of the most common morphological types of Magellanic PNs, from the broad-band images in our surveys. These PNs are more than 50 times farther away than the typical galactic PNs, yet the major morphological features are easily recognized, as are the location of their CSs, when visible.

In the following sections we describe some of the most important results that we obtain from the analysis of our PN samples.

\subsection{Misclassified planetary nebulae}

Extra-Galactic PNs in general, and Magellanic PNs in particular, are typically discovered with $\mathrm{H} \alpha$ or [OIII] $\lambda 5007$ surveys, with the on- and off-band observations technique. The PN nature of the [OIII] $\lambda 5007$-bright object can be generally confirmed by means of spectroscopic follow-up. Marginal misclassification is possible even after spectroscopic 
TABLE 2. Morphological distribution

$\begin{array}{lll}\text { Morphological type } & \text { \% LMC } & \text { \% SMC } \\ & & \\ \text { Round (R) } & 29 & 35 \\ \text { Elliptical (E) } & 17 & 29 \\ \text { R+E (symm.) } & 46 & 64 \\ \text { Bipolar (B) } & 34 & 6 \\ \text { Bipolar core (BC) } & 17 & 24 \\ \text { B+BC (asymm.) } & 51 & 30 \\ \text { Point-symmetric (P) } & 3 & 6\end{array}$

analysis, and only the spatially resolved observations can ultimately confirm the PN nature.

Among the 230 LMC PNs known, about 80 have been observed with HST by us, and another 30 had been observed earlier by Dopita and Blades. In the SMC, we acquired $H S T$ observations of 29 targets, of the 55 known SMC PNs. The spatially resolved sample consists of the [OIII] $\lambda 5007$ bright half of the known Magellanic PNs, given that we had selected the brightest PNs in order to fit the observations within one HST orbit. Thus our sample is representative of the (bright) Magellanic PNs.

It is then extremely interesting to note that we confirmed $100 \%$ of the LMC targets to be PNs, while $10 \%$ of the SMC targets were, instead, ultra-compact H II regions around very young star clusters (Stanghellini, Villaver, Shaw, \& Mutchler 2003b). This result bears on the validity of the PN luminosity function (PNLF) as a secondary distance scale indicator. In fact, the extra-Galactic PNLF is populated by the [OIII] bright PNs of each studied population, and our result seems to indicate that such populations may be contaminated by H II regions in different ways depending on the galaxian metallicity.

\subsection{PN morphology}

The morphology of Galactic PNs has been studied rather thoroughly in the past decade, and it has been found that the morphological types correlate with the PN progenitor's evolutionary history, and the stellar mass. There is strong evidence that asymmetric (e. g., bipolar) PNs are the progeny of the massive AGB progenitors (3-8 $\left.\mathrm{M}_{\odot}\right)$. Bipolar PNs are nitrogen enriched and carbon poor (Stanghellini, Villaver, Manchado, \& Guerrero $2002 b)$. The analysis of the morphological types and their distribution in a PN population is then very useful to infer the age and history of a given stellar sample.

Galactic PNs have been classified as round, elliptical, bipolar (and quadrupolar), bipolar core (those bipolar PNs whose lobes are too faint to be detected, but whose equatorial ring is very evident), and point-symmetric. The majority of Galactic PNs are elliptical, but the actual number of bipolars could be underestimated, given that they typically lie in the Galactic plane (i.e., they may suffer high reddening). PNs in the Clouds, when spatially resolved, show the same admixture of morphological types than the Galactic PNs (see Fig. 1). While we do not attempt a statistical comparison of the MC and Galactic PN morphological types, given the selection effects that hamper Galactic PNs, we can meaningfully compare the LMC and SMC samples. Both samples suffer from low field extinction, and they have been preselected in more or less the same way.

The results of the morphological distribution in the Clouds is summarized in Table 2. Together with the percentage in each morphological class, we give the total of symmetric (round and elliptical) and asymmetric (bipolar and bipolar core) PNs. One striking dif- 
ference between the two distributions is that the fraction of bipolar PNs in the LMC is almost six times that of the SMC. Bipolar PNs are easily recognized, thus this is a sound result. If we add to the asymmetric PN count the bipolar core PNs, we obtain that half of the LMC PNs are asymmetric, while only a third of the SMC PNs are asymmetric. Observational biases play in the same way for the two samples.

What insight can we get from the morphological results? First of all, it is clear that the set of processes that are involved in the formation of the different PN shapes are at work in all galaxies where morphology has been studied. Second, the SMC environment may disfavor the onset of bipolarity in PNs. Otherwise, the different morphological statistics may indicate different populations of stellar progenitors in the two Clouds. While it seems reasonable to conclude that a low metallicity environment is unfavorable to bipolar evolution, the exact causes have not been studied yet. A detailed study of metallicity and mass loss may clarify this point. On the other hand, the different morphological statistics may simply be related to a lower average stellar mass of the PN progenitors in the SMC. If this was the case, we should observe also lower CS masses in the SMC PNs than in the LMC PNs. Our preliminary measurements seem to indicate that this is also the case (Villaver, Stanghellini, \& Shaw, in preparation).

\subsection{Surface brightness evolution}

The surface brightness of LMC and SMC PNs correlates with the photometric radius, as illustrated in Figure 2. In the figure we plot the logarithmic surface brightness, derived from the observed total line fluxes and the apparent radii, versus the photometric radii of the nebulae, measured as the distance from the CS (or the geometrical center of the nebulae) where the enclosed flux is $85 \%$ of the total nebular flux (see Stanghellini et al. 1999). The six panels illustrate the surface brightness- radius relation for the major six emission lines: [OIII] $\lambda 5007, \mathrm{H} \alpha, \mathrm{H} \beta$, [NII] $\lambda 6584$, [OI] $\lambda 6300$, and [SIII] $\lambda 6312$.

The surface brightness- photometric radius relation is tight in all spectral lines, with the exception of the $[\mathrm{NII}]$ emission line, where a larger spread is present, particularly for bipolar PNs. A possible factor is the larger range of nitrogen abundances in bipolar and BC PNs. The surface brightness- photometric radius relations hold only in the cases in which the nebular density $N_{\mathrm{e}}$ is smaller than the critical density, $N_{\text {crit }}$ (the density at which the collisional de-excitation rate balances the radiative transition rate).

A good eye-fit to the surface brightness- photometric radius relation is $\mathrm{SB} \propto \mathrm{R}_{\mathrm{phot}}^{-3}$. This relation can be reproduced via hydrodynamic modeling of evolving PNs and their CSs (Villaver \& Stanghellini, in preparation). The surface brightness- photometric radius relation in the light of $\mathrm{H} \alpha$ (or $\mathrm{H} \beta$ ) is tight enough that it can be used to set the distance scale for Galactic PNs with intrinsic uncertainties of the order of $30 \%$ or less (Stanghellini et al. in preparation), while the current calibration of the Galactic PN distance scales carry errors of the order of $50 \%$ or more.

In Figure 2 we note that the symmetric (round and elliptical) PNs tend to cluster at high surface brightness and low radii, while the asymmetric PNs occupy the lower right parts of the diagrams. This separation can be interpreted with a slower evolutionary rate for the symmetric PNs, which agrees with the idea that symmetric PNs derive from lower mass progenitors (Stanghellini, Corradi, \& Schwarz 1993, Stanghellini et al. 2002b).

\subsection{The [OIII] /H $\beta$ distribution}

In Figure 3 we plot a histogram of the ratio of (reddening-corrected) fluxes of the [OIII] $\lambda 5007$ and $\mathrm{H} \beta$ lines for the PNs of the SMC and the LMC. The median of the SMC distribution is a factor of two lower than for the corresponding LMC distribution ( 


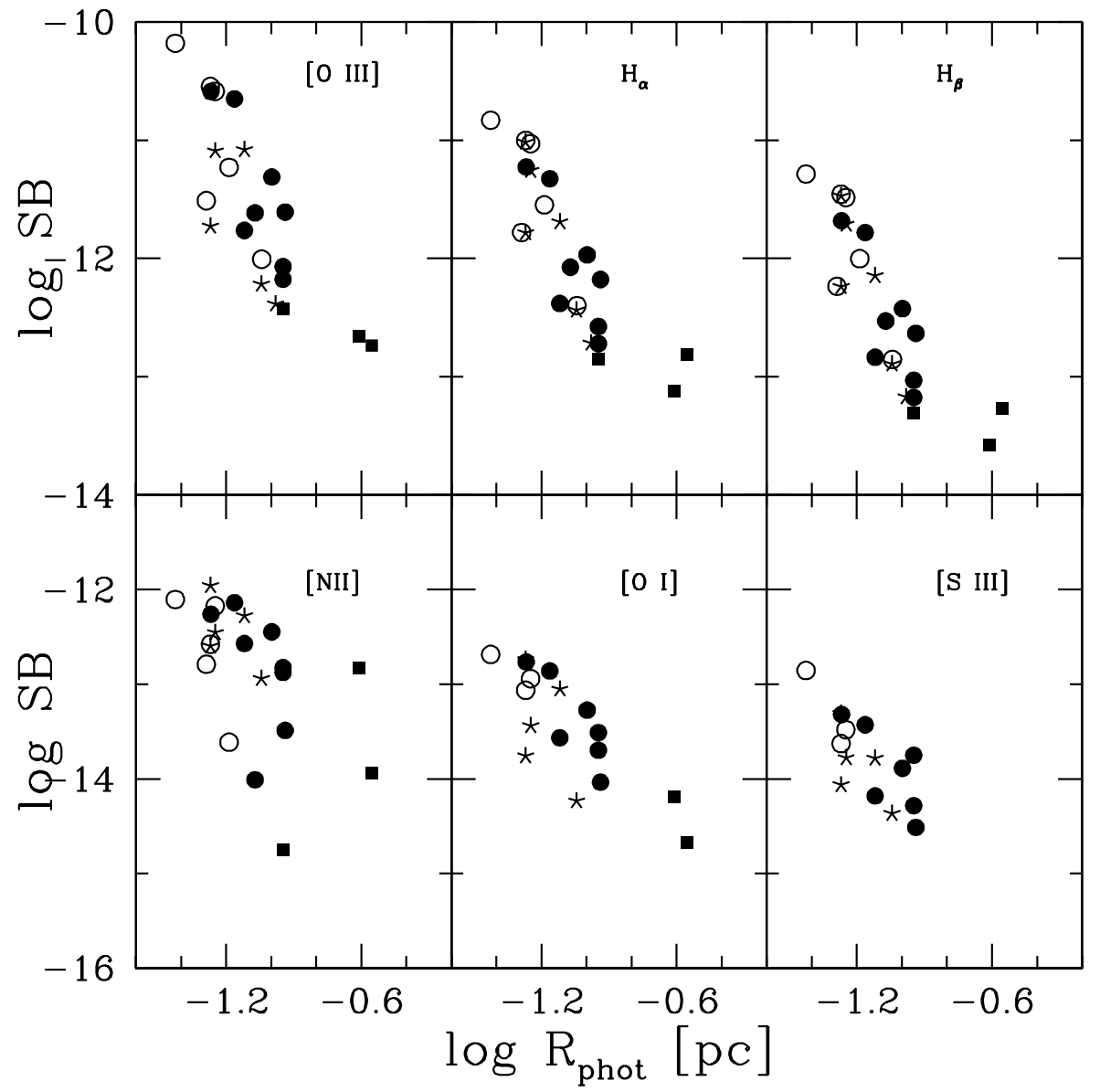

FiguRE 2. Surface brightness decline from the HST multiwavelength images of the LMC and SMC PNs (adapted from Stanghellini et al. 2002a). Emission lines in which the SB is derived are indicated in the panels (see also text). Symbols indicate morphological types: round (open circles), elliptical (asterisks), bipolar core (filled circles), and bipolar and quadrupolar (filled squares). The photometric radii are measured from the [OІІІ] $\lambda 5007$ images, where available.

$<$ [OIII $] / \mathrm{H} \beta>_{\mathrm{SMC}}=5.7 \pm 2.5$ and $\left.<[\mathrm{OIII}] / \mathrm{H} \beta>_{\mathrm{LMC}}=9.4 \pm 3.1\right)$. This result is free of object selection biases since both sets of targets were chosen in much the same way.

The [OIII] / $\mathrm{H} \beta$ emissivity ratio is physically scaled linearly with the $\mathrm{O} / \mathrm{H}$ abundance and the fractional ionization of $\mathrm{O}^{++}$. Also it depends exponentially on the local electron excitation temperature, $\mathrm{T}_{e}\left(\mathrm{O}^{++}\right)$since electron collisions on the high-energy tail of the free energy distribution excite the transition. Of course, $\mathrm{T}_{e}\left(\mathrm{O}^{++}\right)$depends on $\mathrm{O} / \mathrm{H}$ and $\mathrm{O}^{++} / \mathrm{O}$ as well. So interpreting the differences between the [OIII] $/ \mathrm{H} \beta$ ratios of the SMC and the LMC is best done using ionization models.

Our Cloudy (Ferland 1996) models explore the major line emission in a set of Galactic, LMC, and SMC models with same gas density $\left(1000 \mathrm{~cm}^{-3}\right)$ and different metallicities, adequately chosen to represent the average nebula in each studied galaxy, as explained in Stanghellini et al. (2003a). The stellar ionizing spectrum is assumed to be a blackbody with temperatures and luminosities from the H-burning evolutionary tracks for the appropriate galaxian population by Vassiliadis and Wood (1994).

In Figure 4 we show the line intensity relative to $\mathrm{H} \beta$ for the major coolants in the SMC, 


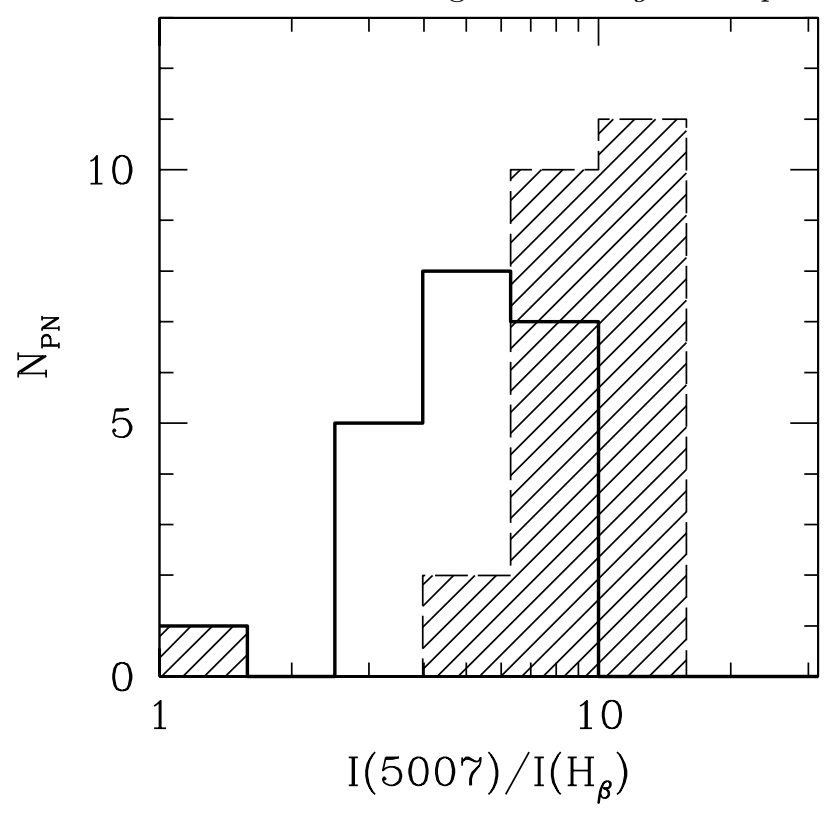

Figure 3. Distribution of the [OIII] $\lambda 5007$ over $\mathrm{H} \beta$ intensity ratios in the SMC (thick histogram) and LMC (shaded histogram) PNs, from Stanghellini et al. 2003a.

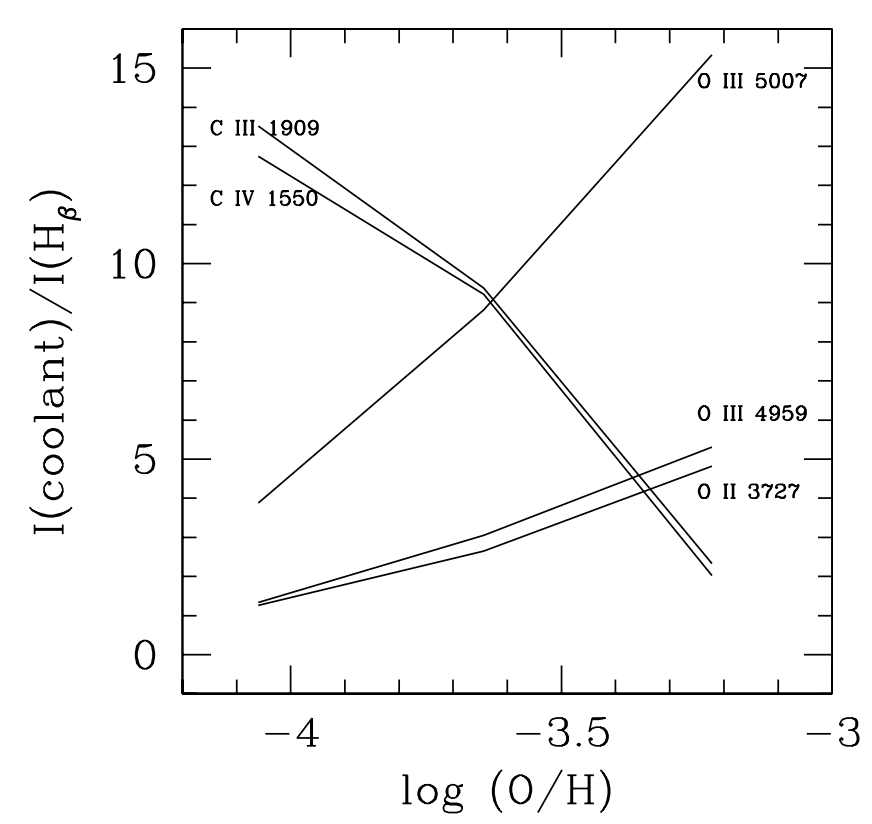

FiguRE 4. Intensity ratios of the major $\mathrm{PN}$ coolants over $\mathrm{H} \beta$, versus oxygen abundances (from Stanghellini et al. 2003a).

LMC, and Galactic PNs, versus the oxygen abundance, as derived from our simplified Cloudy models. While we have calculated the evolution of these intensity ratios following the evolution of the CS from the early post-AGB phase to the white dwarf stage, we only plot here the flux ratios corresponding to the models with the highest temperature, for each PN composition. In general, our target selection tends to favor targets with hottest 
CSs: $\mathrm{T}_{\text {eff }} \geqslant 50,000 \mathrm{~K}$ both in the SMC and in the LMC, thus the set of high-temperature models is the most adequate to reproduce the observations for LMC and SMC PNs.

The cooling processes that determine $\mathrm{T}_{e}\left(\mathrm{O}^{++}\right)$in the SMC, LMC and Galactic PNs are noteworthy. In the Galaxy the primary coolants of PNs with hot CSs are the optical forbidden lines of [OIII] $\lambda 5007$ and other lines of $\mathrm{O}^{+}$and $\mathrm{O}^{++}$. However, in environments in which $\mathrm{O} / \mathrm{H}$ is as low as in the SMC, the primary coolants may become ultraviolet intercombination lines of $\mathrm{C}^{+}$and $\mathrm{C}^{++}$. The simple models described here seem to reproduce very well the optical flux ratios of PNs in the Magellanic Clouds. It will be interesting to confirm these predictions with future UV observations.

\subsection{Chemical analysis of $L M C P N s$}

Studies of Galactic PNs have shown that the bipolar and, more in general, asymmetric PNs present lower carbon abundance (and higher nitrogen abundance) than their symmetric (round or elliptical) counterparts (Peimbert 1978; Stanghellini et al. 2002b). The reason for the carbon underabundance is to be found in late phases of AGB evolution, when the nuclear burning extends to the bottom of the stellar envelope (HBB, see Iben \& Renzini 1983). The result seem to imply that asymmetric PNs are the progeny of the more massive stars $\left(\mathrm{M}>3 \mathrm{M}_{\odot}\right)$ in the PN progenitor range, since only these stars undergo the HBB process (for the yields of the LMC AGB stars see Van den Hoek \& Groenevegen 1997). A statistically sound result is not possible in the Galactic environment, since the asymmetric PNs tend to be located near the Galactic plane, thus their observation may be severely affected by the Galactic plane reddening. With our LMC observations we are in the position to test these findings by circumventing the selection effects. We found that carbon abundance is higher than $1 \times 10^{-4}$ for all round and elliptical PNs, while it can be as low as $\approx 3 \times 10^{-6}$ for the asymmetric PNs (Stanghellini, Shaw, Balick, \& Blades 2000, all abundances are by number, versus hydrogen), in agreement with the trends that were expected form the Galactic results.

We have also studied the abundance-versus-morphology relations for the elements whose abundance do not dramatically change during the evolution of the PN progenitors, such as sulfur, argon, oxygen, and neon (the so-called alpha elements). In Figure 5 we show the relations between neon and, from the top panel to the lower panel, oxygen, argon, and sulfur abundances. The LMC PNs plotted are coded for morphological type, as described in the label. The large circled symbols represent the average LMC abundance of the given elements in the H II regions. We see that there is morphological discrimination between the high and low alpha-element abundance PNs.

\subsection{Central stars of $L M C P N s$}

Our HST programs allowed, for the first time, the direct observation of CSs of extraGalactic PNs. While the observations in slitless mode were specially crafted to detect the nebulae in the most prominent emission lines, the broad-band imagery showed the CSs in approximately $60 \%$ of the targets.

We have measured magnitudes via aperture photometry of the CSs in the LMC (Villaver, Stanghellini, \& Shaw 2003) and SMC PNs. By means of the Zanstra analysis, and using the most recent, uniformly selected He II $\lambda 4686$ fluxes, we have determined the (H I and He II) Zantsra stellar temperatures. Finally, by placing the stars on the log $\mathrm{L}-\log \mathrm{T}_{\text {eff }}$ plane, and comparing their loci with the theoretical evolutionary post-AGB tracks by Vassiliadis \& Wood (1994) we have determine the stellar mass.

The median mass of LMC and SMC CSs, respectively 0.63 and $0.59 \mathrm{M}_{\odot}$, do not differ very much, indicating that the possible variance with metallicity of the factors at play 


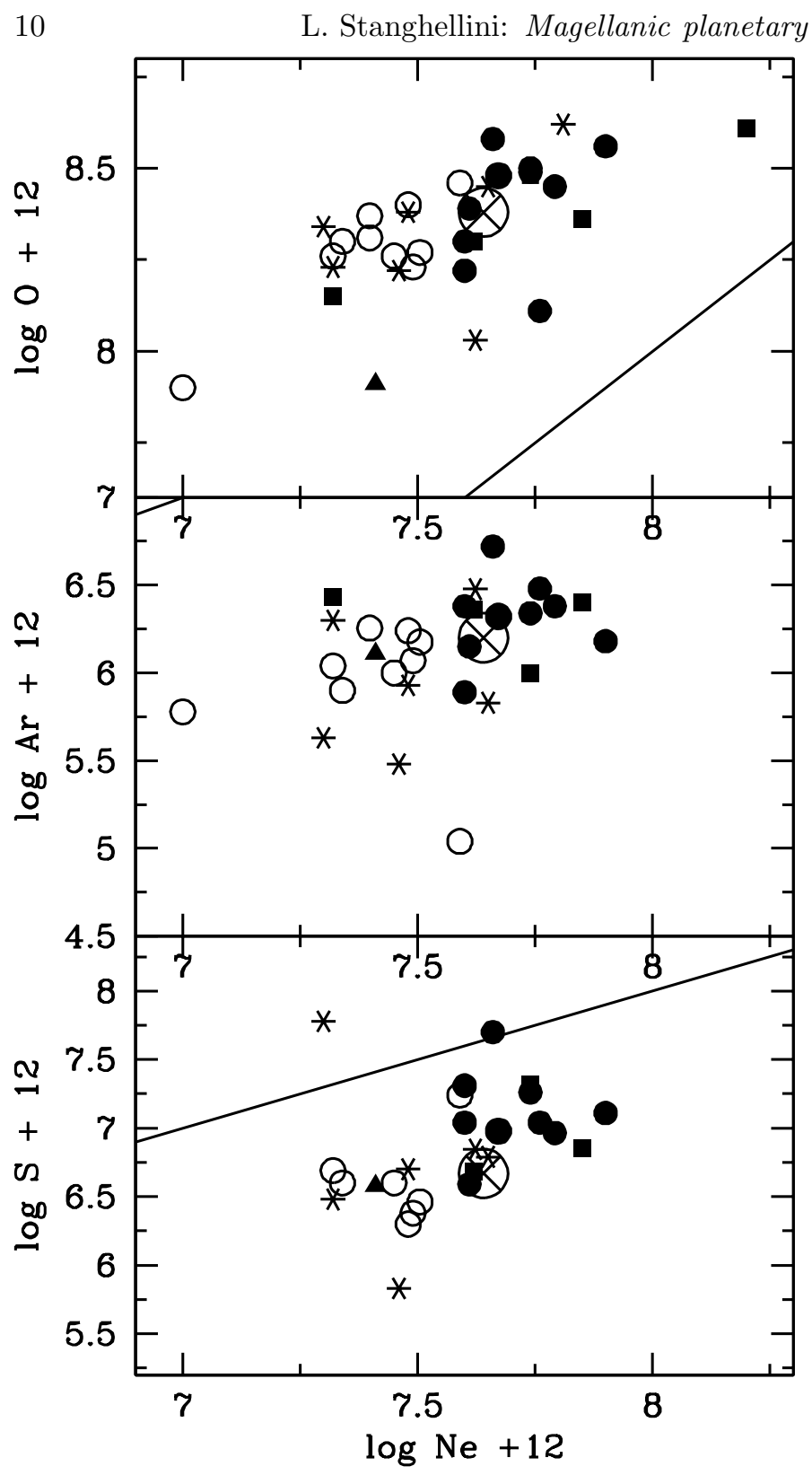

Figure 5. O, Ar, and S vs. Ne abundances of LMC PNs for morphological types round (open circles), elliptical (asterisks), quadrupolar (filled triangles), bipolar core (filled circles), and bipolar (filled squares). The large crossed circle represents the average for LMC H II regions (adapted from Stanghellini et al. 2000).

(initial mass function, star formation rate and history, and mass-loss rate) probably average each other out (Villaver, Stanghellini, \& Shaw, in preparation).

The total number of CSs analyzed so far, whose masses are determined with confidence, is small (approximately 25 objects total). We will examine a larger sample of CSs in the Clouds to deepen the analysis of possible correlations between the core mass and the morphology of PNs in the future.

Knowing the position of the CSs on the $\log \mathrm{L}-\log \mathrm{T}_{\text {eff }}$ diagram is essential not 
only for the mass determination, but also to constrain the transition time (time interval between the quenching of the envelope ejection at the AGB tip and the PN illumination, see Stanghellini \& Renzini 2000). Stellar evolutionary models for post-AGB stars start with ad hoc models that imply strong (or semi-empirical) assumptions on the mass loss rate. From an observational viewpoint, the timing of post-AGB evolution is derived from the dynamic time of the PN. For Galactic PNs the comparison of the dynamic and evolutionary time-scales has the double offset of the (undetermined) transition time and the distance bias, while for LMC and SMC PNs the difference between the observed and theoretical evolutionary time allows the estimate of the transition time.

The transition time is relevant in post-AGB population synthesis, for example in the studies of the PNLF as a secondary distance-scale indicator, and also in studies concerning the UV contribution from post-AGB stars in galaxies. Further constraints on the effective temperature of the CSs derived from the UV continuum fitting, from our Cycle 10 UV spectra, will strengthen the results on stellar evolution.

\section{Summary}

Magellanic PNs are ideal probes to study stellar evolution and populations of lowand intermediate-mass stars. The use of the HST is fundamental for determining the PN shapes, the radii, and also to detect the CSs. Furthermore, only with the use of spatially resolved images one can identify the LMC and SMC PNs unambiguously, without the accidental inclusion of compact $\mathrm{H}$ II regions in the PN samples.

We have presented the results derived from our HST programs, in the background of the important work that has been done in LMC and SMC PNs previously.

We found that PNs have the same morphological types in the Galaxy, the LMC, and the SMC. We also found that the distribution of the morphological types is noticeably different in the SMC and the LMC, and that the LMC seems to be populated by PNs whose progenitors are, on average, more massive.

We analyzed morphology and chemical composition of a sample of LMC PNs, and found that asymmetric (bipolar and bipolar core) PNs have higher Ne, Ar, and S abundance than symmetric (round and elliptical) PNs in the LMC, a confirmation that they trace more recent stellar populations.

An empirical relation between the nebular radii and the surface brightness is found to hold in both SMC and LMC PNs, independent of morphological type. The relation, once calibrated, will be used to determine the distance scale for Galactic extended PNs.

The PN cooling is affected by metallicity, and it seems that the [OIII] $\lambda 5007$ emission is not always the ideal line to detect bright PNs in all Galaxies, since the strongest cooling lines in very low metallicity PNs seem to be the UV [CIII] (and [CIV] ) semiforbidden emission.

The observed Magellanic CSs that we discuss here constitute the first sizable sample of CS beyond the Milky Way that has been directly observed. While we found only marginal differences between the LMC and the SMC median CSs masses of the CSs, we need to enlarge the sample of CS whose masses can be reliably measured, given the importance of knowing initial-to final-mass relation in different metallicity environments.

The work presented in $\S 4$ has been developed in collaboration with Dick Shaw, Eva Villaver, Bruce Balick, and Chris Blades. 
Aller, L. H. 1961, AJ, 66, 37

Aller, L. H., Keyes, C. D., Maran, S. P., Gull, T. R., Michalitsianos, A. G., \& Stecher, T. P. 1987, ApJ, 320, 159

Barlow, M. J. 1989, IAU Symp. 131: Planetary Nebulae, 131, 319

Blades, J. C. et al. 1992, ApJ, 398, L41

Boroson, T. A. \& Liebert, J. 1989, ApJ, 339, 844

Costa, R. D. D., de Freitas Pacheco, J. A., \& Idiart, T. P. 2000, A\&AS, 145, 467

Dopita, M. A. et al. 1996, ApJ, 460, 320

Egan, M. P., Van Dyk, S. D., \& Price, S. D. 2001, AJ, 122, 1844

Feast, M. W. 1968, IAU Symp. 34: Planetary Nebulae, 34, 34

Iben, I., Jr., \& Renzini, A. 1983, ARA\&A, 21, 271

Kaler, J. B., \& Jacoby, G. H. 1991, ApJ 382, 134

Leisy, P. \& Dennefeld, M. 1996, A\&AS, 116, 95

Leisy, P., Dennefeld, M., Alard, C., \& Guibert, J. 1997, A\&AS, 121, 407

Lindsay, E. M. 1955, MNRAS, 115, 241

Meatheringham, S. J. Dopita, M. A. 1991a, ApJS, 75, 407

Meatheringham, S. J. \& Dopita, M. A. 1991b, ApJS, 76, 1085

Ferland, G. J. 1996, Hazy, a Brief Introduction to Cloudy, University of Kentucky Dept. of Physics and Astronomy Internal Report

Meyssonnier, N. \& Azzopardi, M. 1993, A\&AS, 102, 451

Morgan, D. H. 1998, Publications of the Astronomical Society of Australia, 15, 123

Murphy, M. T. \& Bessell, M. S. 2000, MNRAS, 311, 741

Parker, Q. A. \& Phillipps, S. 1998, Publications of the Astronomical Society of Australia, 15, 28

Peimbert, M. 1978, in IAU Symp. 76, Planetary Nebulae, ed. Y. Terzian (Dordrecht: Reidel), 215

Peimbert, M. 1984, IAU Symp. 108: Structure and Evolution of the Magellanic Clouds, 108, 363

Peimbert, M. 1997, in IAU Symp. 180, Planetary Nebulae, ed. H. Habing \& H. Lamers (Dordrecht: Kluwer), 175

Shaw, R. A., Stanghellini, L., Mutchler, M., Balick, B., \& Blades, J. C. 2001, ApJ, 548, 727 (Paper I)

Smith, R. C. et al. 1996, American Astronomical Society Meeting, 188, 5101

Stanghellini, L., Corradi, R. L. M., \& Schwarz, H. E. 1993, A\&A, 279, 521

Stanghellini, L., Blades, J. C., Osmer, S. J., Barlow, M. J., \& Liu, X.-W. 1999, ApJ, 510, 687

Stanghellini, L., Shaw, R. A., Balick, B., \& Blades, J. C. 2000, ApJ, 534, L167

Stanghellini, L., Shaw, R. A., Mutchler, M., Palen, S., Balick, B., \& Blades, J. C. 2002a, ApJ, 575, 178 (Paper II)

Stanghellini, L., Villaver, E., Manchado, A., \& Guerrero, M. A. 2002b, ApJ, 576, 285

Stanghellini, L., Shaw, R. A., Balick, B., Mutchler, M., Blades, J. C., \& Villaver, E. 2003a, ApJ, in press (Paper III)

Stanghellini, L., Villaver, E., Shaw, R. A., \& Mutchler, M. 2003b, ApJ, in press (Paper IV)

van den Hoek, L. B. \& Groenewegen, M. A. T. 1997, A\&AS, 123, 305

Vassiliadis, E., Dopita, M. A., Morgan, D. H., \& Bell, J. F. 1992, ApJS, 83, 87

Vassiliadis, E. \& Wood, P. R. 1994, ApJS, 92, 125

Vassiliadis, E. et al. 1998, ApJ, 503, 253

Villaver, E., Stanghellini, L., \& Shaw, R. A. 2003, ApJ, in press

Webster, B. L. 1969, MNRAS, 143, 113

Webster, B. L. 1978, IAU Symp. 76: Planetary Nebulae, 76, 11

Westerlund, B. E. 1964, IAU Symp. 20: The Galaxy and the Magellanic Clouds, 20, 316W

Westerlund, B. E. 1968, IAU Symp. 34: Planetary Nebulae, 34, 23 